\title{
Octave-spanning coherent supercontinuum generation in an AIGaAs-on-insulator waveguide
}

Kuyken, Bart; Billet, Maximilien; Leo, Francois; Yvind, Kresten; Pu, Minhao

Published in:

Optics Letters

Link to article, DOI:

10.1364/ol.45.000603

Publication date:

2020

Document Version

Peer reviewed version

Link back to DTU Orbit

Citation (APA):

Kuyken, B., Billet, M., Leo, F., Yvind, K., \& Pu, M. (2020). Octave-spanning coherent supercontinuum generation in an AlGaAs-on-insulator waveguide. Optics Letters, 45(3), 603-606. https://doi.org/10.1364/ol.45.000603

\section{General rights}

Copyright and moral rights for the publications made accessible in the public portal are retained by the authors and/or other copyright owners and it is a condition of accessing publications that users recognise and abide by the legal requirements associated with these rights.

- Users may download and print one copy of any publication from the public portal for the purpose of private study or research.

- You may not further distribute the material or use it for any profit-making activity or commercial gain

- You may freely distribute the URL identifying the publication in the public portal 


\section{Octave-spanning coherent supercontinuum generation in an AlGaAs-on- insulator waveguide}

Preprint · January 2020

CITATIONS

0

5 authors, including:

Bart Kuyken

Ghent University

165 PUBLICATIONS 3,154 CITATIONS

SEE PROFILE

Maximilien Billet

Ghent University

42 PUBLICATIONS 116 CITATIONS

SEE PROFILE

Some of the authors of this publication are also working on these related projects:

Nanophotonics View project

Integrated Nonlinear Optics - AlGaAs-On-Insulator Nonlinear Photonics View project
READS

144

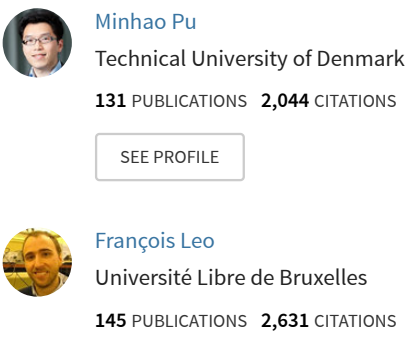

SEE PROFILE 
To be published in Optics Letters:

Title: $\quad$ Octave-spanning coherent supercontinuum generation in an AIGaAs-on-insulator wavegu Authors: $\quad$ Bart Kuyken,Minhao Pu,Maximilien Billet,François Leo,Kresten Yvind Accepted: 21 November 19

Posted 22 November 19

Doc. ID: $\quad 379426$ 


\title{
Octave-spanning coherent supercontinuum generation in an AlGaAs-on-insulator waveguide
}

\author{
Bart Kuyken, ${ }^{1,2}$ Maximilien Billet, ${ }^{1,2,3}$ Francois Leo, ${ }^{3}$ Kresten YVInd, ${ }^{4}$ AND \\ MiNHAO Pu ${ }^{4, *}$ \\ ${ }^{1}$ Photonics Research Group, Department of Information Technology, Ghent University IMEC, Ghent B-9000, Belgium \\ ${ }^{2}$ Center for Nano- and Biophotonics (NB-Photonics), Ghent University, Ghent, Belgium \\ ${ }^{3}$ OPERA-Photonique, Université libre de Bruxelles (ULB), 50 Av. F. D. Roosevelt, CP 194/5, B-1050 Bruxelles, Belgium \\ ${ }^{4}$ DTU Fotonik, Department of Photonics Engineering, Technical University of Denmark, Building 343, DK-2800 Kgs. Lyngby, Denmark \\ *Corresponding author: mipu@fotonik.dtu.dk
}

Received XX Month XXXX; revised XX Month, XXXX; accepted XX Month XXXX; posted XX Month XXXX (Doc. ID XXXXX); published XX Month XXXX

We demonstrate supercontinuum generation over an octave spaning from 1055 to $2155 \mathrm{~nm}$ on the highly nonlinear aluminum gallium arsenide-on-insulator (AlGaAsOI) platform. This is enabled by the generation of two dispersive waves in a 3-mm-long dispersionengineered nano-waveguide. The waveguide is pumped at telecom wavelengths $(1555 \mathrm{~nm})$ with $3.6 \mathrm{pJ}$ femtosecond pulses. We experimentally validate the coherence of the generated supercontinuum around the pump wavelength $(1450-1750 \mathrm{~nm})$, and our numerical simulation shows a high degree of coherence over the full spectrum. (C) 2018 Optical Society of America

http://dx.doi.org/10.1364/OL.99.099999

Supercontinuum generation (SCG), first demonstrated in bulk by Alfano and Shapiro in 1970 [1], is one of the most efficient ways to obtain spatially and often temporally coherent broadband light sources. It has found its way to many applications including metrology [2], telecommunication [3], spectroscopy [4] and short pulse sciences [5]. With the advent of photonic crystal fibers and their potential for tuning the dispersion, the research field has undergone enormous progress in the last 20 years [6]. More recently, integrated photonic platforms have been widely used for SCG because they potentially offer a compact, low-cost solution for SCG. Various material platforms including silicon [7], [8], chalcogenide (ChG) [9], [10], lithium niobate [11], [12] and silicon nitride $\left(\mathrm{Si}_{3} \mathrm{~N}_{4}\right)$ [13], [14], have been utilized to realize broadband SCG.

Generation of an octave-spanning supercontinuum (SC) relies on third-order $\left(\chi^{(3)}\right)$ nonlinear effects. The generation of octave spanning coherent SCG is essential for stabilizing frequency combs through $f$-to- $2 f$ interferometers and consequent self-referencing. This is crucial in frequency metrology and precision spectroscopy [15] applications. Apart from SCG, second harmonic generation
(SHG), based on the quadratic $\left(\chi^{(2)}\right)$ nonlinear process, is also required in an $f$-to- $2 f$ interferometer. However, because of the centrosymmetric crystal structures, materials such as silicon and $\mathrm{Si}_{3} \mathrm{~N}_{4}$ lack intrinsic $\chi^{(2)}$ effect, which inhibits the integration of SC source and second harmonic generator in a single material platform. Although significant progress has been made to realize effective $\chi^{(2)}$ process in silicon [16] and $\mathrm{Si}_{3} \mathrm{~N}_{4}$ [17], the achieved efficiency have been limited hampering on-chip $f$-to- $2 f$ interferometry. Therefore, it is highly desirable that octavespanning SCG is realized in materials that exhibit both strong intrinsic $\chi^{(2)}$ and $\chi^{(3)}$ nonlinearities. Those materials include aluminum nitride (AlN) [18], gallium nitride (GaN) [19], [20], silicon carbide ( $\mathrm{SiC})$ [21], lithium niobate $\left(\mathrm{LiNbO}_{3}\right)$ [11], [12] [22] and (indium) gallium phosphide ((In)GaP) [23], [24],. Among those materials, aluminum gallium arsenide (AlGaAs) has the strongest intrinsic Kerr coefficient (a nonlinear index of $10^{-17} \mathrm{~m}^{2} \mathrm{~W}$ 1) [25] and a strong quadratic nonlinear coefficient ( $120 \mathrm{pm} / \mathrm{v})$ [26]. Combining the strong material nonlinearity and high light confinement, we have developed the AlGaAs-on-insulator (AlGaAsOI) platform and demonstrated ultra-high effective Kerr nonlinearity of the platform [27]. Moreover, the bandgap of AlGaAs material can be engineered to mitigate the most detrimental nonlinear loss at the telecom wavelength induced by two-photon absorption (TPA) while the optimization of fabrication technology for this high confinement waveguide platform ensures a low linear loss, which leads to a high nonlinear figure of merit (FOM) as a Kerr nonlinear platform [28]. Recently, efficient SHG has also been demonstrated in similar high-confinement (Al)GaAsOI waveguides [29], [30].

Benefiting from the ultra-high effective Kerr nonlinearity, we previously demonstrated efficient generation of a frequency comb covering the telecom C-band based on self-phase modulation (SPM) by pumping an AlGaAsOI waveguide using pico-second pulses with a high repetition rate (at tens of Gigahertz) for highspeed communication transmission system [31]. Very recently, Chiles et al. have realized suspended AlGaAs waveguides and 
demonstrated SCG at both the near-infrared (NIR) and midinfrared (MIR) range by using femtosecond-pulse pumping [32]. In this work, we demonstrate octave-spanning SCG in an AlGaAsOI waveguide in the near-infrared range through dispersive wave generation over an octave at $f$ and $2 f$ frequencies, which is favorable concerning the power distribution in the generated SC for a sufficient $f$-to- $2 f$ self-beating signal [33]. The coherence of the generated SC has also been verified experimentally using an interferometry method around the pump wavelength. Our numerical simulation also shows a high degree of coherence of the SC over the full octave span. Our demonstration shows a huge potential of the AlGaAsOI platform in the realization of chip-scale $f$ to- 2 finterferometry for applications in frequency metrology.

We fabricated waveguides on an AlGaAsOI wafer, where a thin AlGaAs layer on top of a low-index insulator layer resides on a semiconductor substrate. The aluminum composition of the AlGaAs layer is $21 \%$, which corresponds to a bandgap of $\sim 1.72 \mathrm{eV}$. Wafer bonding and substrate removal processes are used to fabricate the AlGaAsOI wafer [34]. Owing to the large index contrast between AlGaAs and silica, light can be confined in a submicron waveguide core. It does not only enhance the device nonlinearity but also enable efficient dispersion engineering, which is essential for SCG. As shown in Fig. 1, the group velocity dispersion (GVD) of the AlGaAsOI waveguide can be engineered from the normal dispersion regime (in the case of bulk AlGaAs) to the anomalous dispersion regime by tailoring its cross-sectional dimension in a sub-micron scale $(300 \mathrm{~nm} \times 500 \mathrm{~nm})$. The waveguide exhibits a low third-order dispersion at the pump wavelength $(1555 \mathrm{~nm})$ and has two zero dispersion wavelengths (ZDWs) between the $f$ and $2 f$ wavelengths, which is potentially suitable for dispersive wave generation at $f$ and $2 f$ wavelengths [6].

The waveguide pattern was defined firstly in the electron-beam resist hydrogen silsesquioxane (HSQ Dow Corning FOX-15) by electron-beam lithography (JEOL JBX-9500FS) [35]. The pattern was then transferred into the AlGaAs layer using a boron trichloride $\left(\mathrm{BCl}_{3}\right)$-based dry etching process in an inductively coupled plasma reactive ion etching (ICP-RIE) machine. A scanning electron microscopy (SEM) picture of the etched waveguide is shown in the inset of Fig. 2 where the simulated field distribution of the fundamental TE mode is superimposed on the image. As the refractive index of HSQ is relatively low (similar to $\mathrm{SiO}_{2}$ ), it was kept on top of the AlGaAs device pattern. Finally, the waveguides were clad in a 3- $\mu$ m-thick silica layer using plasma enhanced chemical vapour deposition (PECVD). The waveguide is inversely

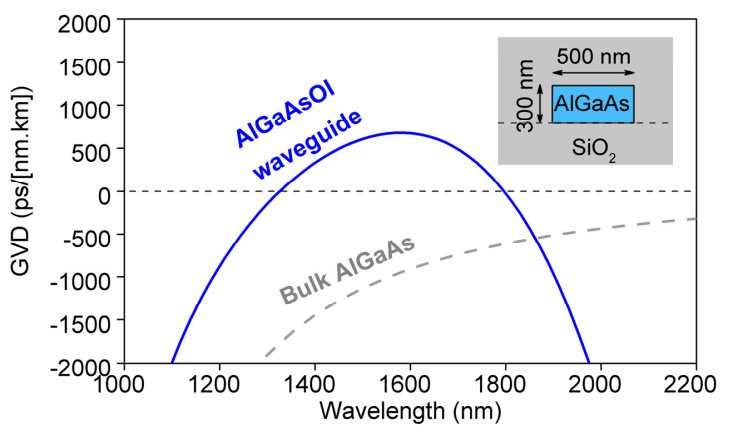

Fig. 1. Calculated group velocity dispersion (GVD) as a function of the wavelength for an AlGaAs-on-insulator (AlGaAsOI) waveguide with the cross-sectional dimension of $300 \mathrm{~nm} \times 500 \mathrm{~nm}$. The grey dash line shows the dispersion for the bulk AlGaAs material.

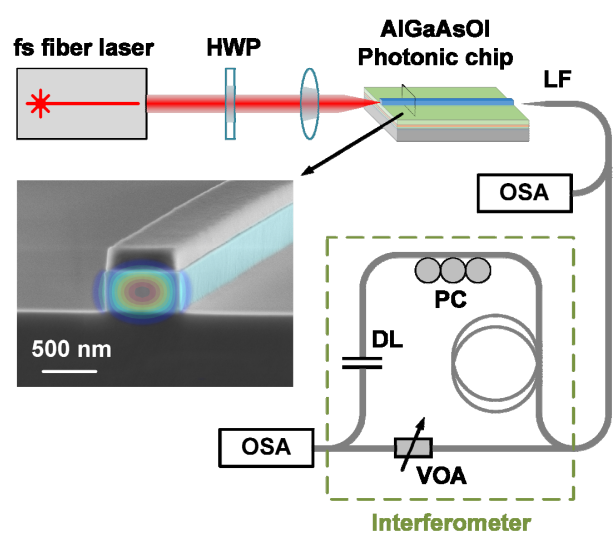

Fig. 2. Experimental setup for supercontinuum generation in an AlGaAsOI waveguide. HWP: half-wave plate; LF: lensed fiber; PC: polarization controller; DL: delay line; VOA: variable optical attenuator. OSA: optical spectrum analyzer. Inset shows a scanning electron microscopy (SEM) picture of the fabricated waveguide before it is been clad in silica.

tapered to $120 \mathrm{~nm}$ at the sample facets for better input and output coupling [36].

The experimental setup is shown in Fig. 2. A femtosecond fiber laser emits 100 -fs pulses with a repetition rate of $90 \mathrm{MHz}$ at $1555 \mathrm{~nm}$. The light is coupled to the photonic chip in free space via a focusing lens with a coupling loss of $12 \mathrm{~dB}$. The half-wave plate (HWP) is used to align the light to the TE-polarization of the waveguide. The output of the chip is coupled to a lensed fiber (LF), and the coupling loss is $4 \mathrm{~dB}$. The propagation loss of the AlGaAsOI waveguide is about $2 \mathrm{~dB} / \mathrm{cm}$ which is extracted from a cut-back measurement. The output spectrum is monitored with an optical spectrum analyzer (OSA) (YOKOGAWA AQ6375B for $>1200 \mathrm{~nm}$ and AND0 AQ6317B for $<1200 \mathrm{~nm}$ ). For a coherence measurement, the output spectrum is recorded after passing through a fiber-based asymmetric Mach-Zehnder interferometer containing a tunable delay (DL) a polarization controller (PC) and a variable attenuator (VOA).

The spectral broadening effects in SCG with femtosecond pulses are dominated by soliton dynamics and the soliton number $N$ is dependent on the peak power $P$ by $N=\sqrt{L_{D} / L_{N L}}$, where $L_{D}=\left(T_{0}^{2}\right) /\left|\beta_{2}\right|$ and $L_{N L}=1 / \gamma P$ are the dispersion length and the nonlinear length, respectively, with $T_{0}$ the full width at half maximum pulse duration of the injected soliton, $\beta_{2}$ the second order dispersion and $\gamma$ the Kerr nonlinear coefficient of the AlGaAsOI waveguide. We characterized the output spectra with a pump peak power range that corresponds to a soliton number from 1 to 24. The measured output spectrum evolution of the SCG is shown in Fig. 3(a). The black curve shows the spectrum of the coupled pump pulses with 50 femtojoules of energy. For clarity, throughout the paper the pulse energies are the on-chip pulse energies. As the pump pulse energy increases, a significant broadening is observed starting from pulse energies of $0.9 \mathrm{pJ}$. Two dispersive waves are present at around $1100 \mathrm{~nm}$ and $2100 \mathrm{~nm}$, respectively, when the pump pulse energy reaches $3.6 \mathrm{pJ}$. The spectrum starts saturating when the pump pulse energy is increased further due to three-photon absorption (ThPA). The 

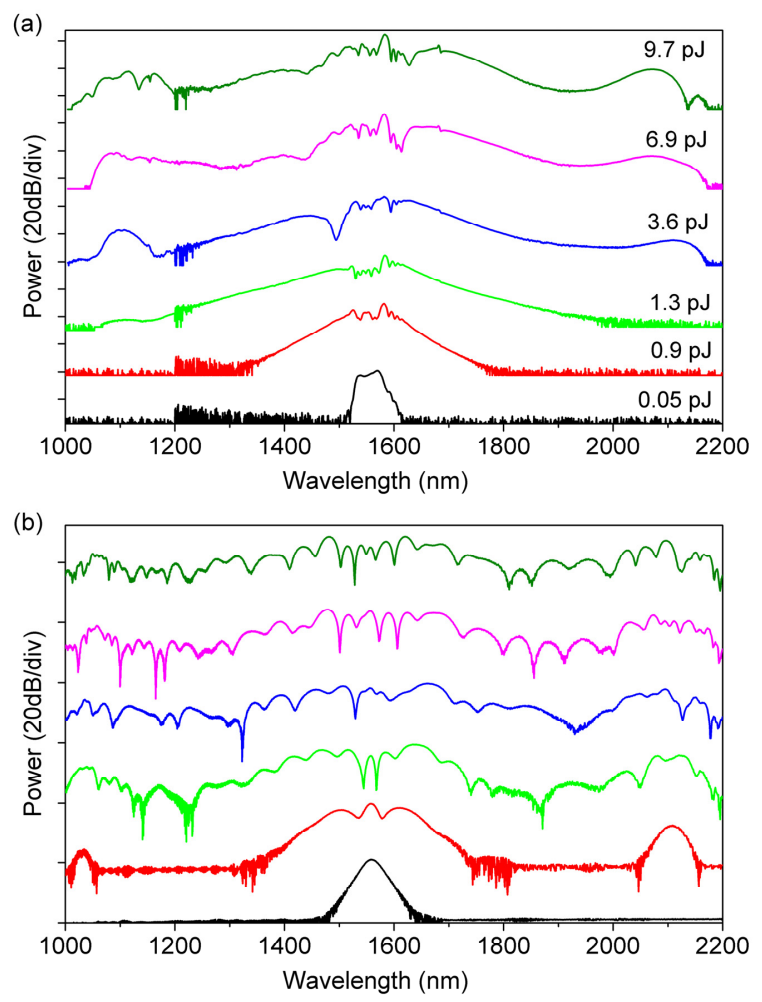

Fig. 3. Output spectra of the 500 -nm wide waveguide for different pump pulse energies. (a) Experimental data and (b) Simulations.

obtained SC has a 40-dB bandwidth of about $1100 \mathrm{~nm}$ covering $1055 \mathrm{~nm}$ to $2155 \mathrm{~nm}$.

To understand the effects involved in the SCG, we model our system with a generalized nonlinear Schrödinger equation (NLSE) taking into account the Kerr nonlinearity $\left(630 \mathrm{~m}^{-1} \mathrm{~W}^{-1}\right)$ of the AlGaAsOI waveguide [28] by using the spilt-step method [37]. The output spectra of the waveguide are then computed as shown in Fig. 3(b). Compared with the experimental data in Fig. 3(a), the spectrum broadening effect is well reproduced by the NLSE. We can identify two distinct positions in the spectra at $1100 \mathrm{~nm}$ and $2100 \mathrm{~nm}$, respectively, where a peak is visible. To associate a physical effect to these two peaks we calculate the predictable frequencies of the generated dispersive waves. The phasematching condition between the soliton centered at a frequency $\omega_{s}$ and a dispersive wave at a frequency $\omega_{D W}$ can be expressed by the following equation [6]

$$
\beta\left(\omega_{D W}\right)=\beta\left(\omega_{s}\right)+\left(\omega_{D W}-\omega_{s}\right) v_{g}^{-1}+\left(1-f_{R}\right) \gamma P
$$

where $\beta$ is the propagation constant of the wave, $v_{g}$ is the group velocity at the pump frequency, $f_{R}$ is the fractional Raman response, $\gamma$ is the nonlinear parameter and $P$ is the peak power of the soliton. By neglecting the nonlinear contribution into the phase-matching condition, we can write the phase mismatch between a wave of frequency $\omega_{s}$ and the dispersive wave [14]

$$
\Delta \beta(\omega)=\beta_{\text {int }}=\beta(\omega)-\beta\left(\omega_{s}\right)-\left(\omega-\omega_{s}\right) v_{g}^{-1}
$$

The phase matching appears when the integrated dispersion $\beta_{\text {int }}$ is equal to zero. Figure 4(a) shows the calculation of $\beta_{\text {int }}$ as a function of the wavelength for the AlGaAsOI waveguide. It is seen that it is possible to achieve the phase-matching condition in the near infrared at $1025 \mathrm{~nm}$, and in the mid infrared at $2130 \mathrm{~nm}$. Figure 4(b) presents an experimental validation of the calculated phase-matching condition by showing the recorded output spectrum of the waveguide pumped by pulses of $6.9 \mathrm{pJ}$ in energy. A good agreement is observed between the calculation and the experiment for the NIR and MIR dispersive wave wavelength positions though a small discrepancy is observed due to the fabrication imperfection as nanometer-scale dimension perturbations may induce the shift of the dispersive waves.

The coherence of the generated SC was investigated theoretically and experimentally. The coherence $g_{12}^{(1)}$ is evaluated by using the following formula [7]:

$$
g_{12}^{(1)}(\lambda)=\frac{\left\langle E_{1}^{*}(\lambda) E_{2}(\lambda)\right\rangle}{\sqrt{\left\langle\left|E_{1}(\lambda)\right|^{2}\right\rangle\left\langle\left|E_{2}(\lambda)\right|^{2}\right\rangle}}
$$

where $E_{1}$ and $E_{2}$ denote spectra computed from NLSE with different initial noise conditions. The input noise consists of adding one photon per mode with a random phase in the initial spectrum. In practice, we performed 20 independent simulations and the coherence is calculated by using Eq. (3) by averaging each pair of simulated output spectra. Figure 5(a) shows the computed coherence of the generated SC obtained at different pump pulse energy. For $9.7 \mathrm{pJ}$ pulse energy, it is seen that the parameter $g_{12}^{(1)}$ has a value close to 1 over the entire spectral range of the SC indicating a strong coherence, which makes it promising in metrology applications that require $f$-to- $2 f$ self-referencing.

The coherence can be estimated experimentally from the fringe visibility $V$ of an interference pattern obtained in an interferometry measurement by using the below equation:

$$
\left|g_{12}^{(1)}(\lambda)\right|=\frac{I_{1}(\lambda)+I_{2}(\lambda)}{2 \sqrt{I_{1}(\lambda) I_{2}(\lambda)}} V(\lambda)
$$

where $I_{1}$ and $I_{2}$ are the intensity from two arms of the interferometer and $V=\left(I_{\max }-I_{\min }\right) /\left(I_{\max }+I_{\min }\right)$, where $I_{\max }$ and $I_{\min }$ correspond to the maxima and minima of each of the fringes. In the interferometer as shown in Fig. 1, the VOA is used to balance the intensities of two arms and the PC is used to align the polarization of the output light from two arms, which ensures a strong
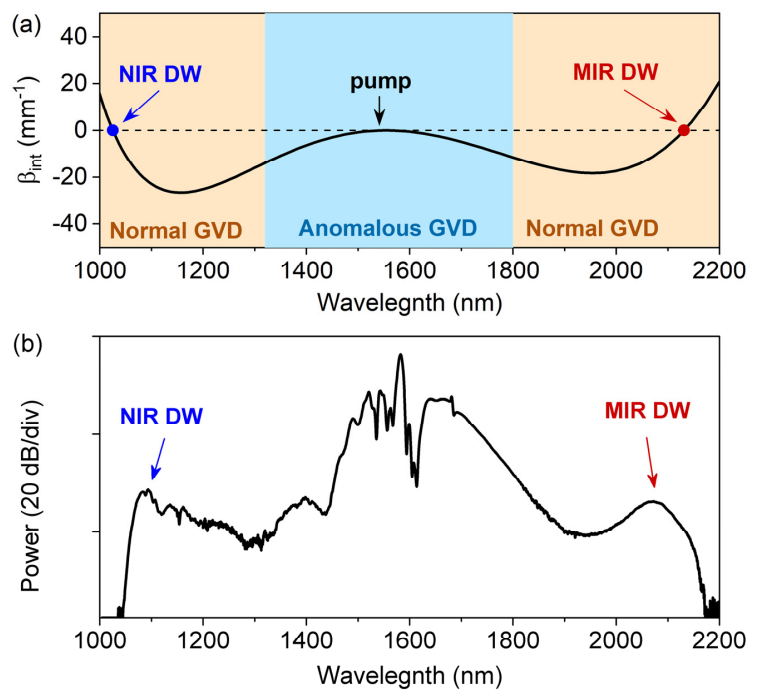

Fig. 4. (a) The calculated integrated dispersion for the AlGaAsOI waveguide. (b) The output spectrum of the AlGaAsOI waveguide with a pump pulse energy of $6.9 \mathrm{pJ}$. 

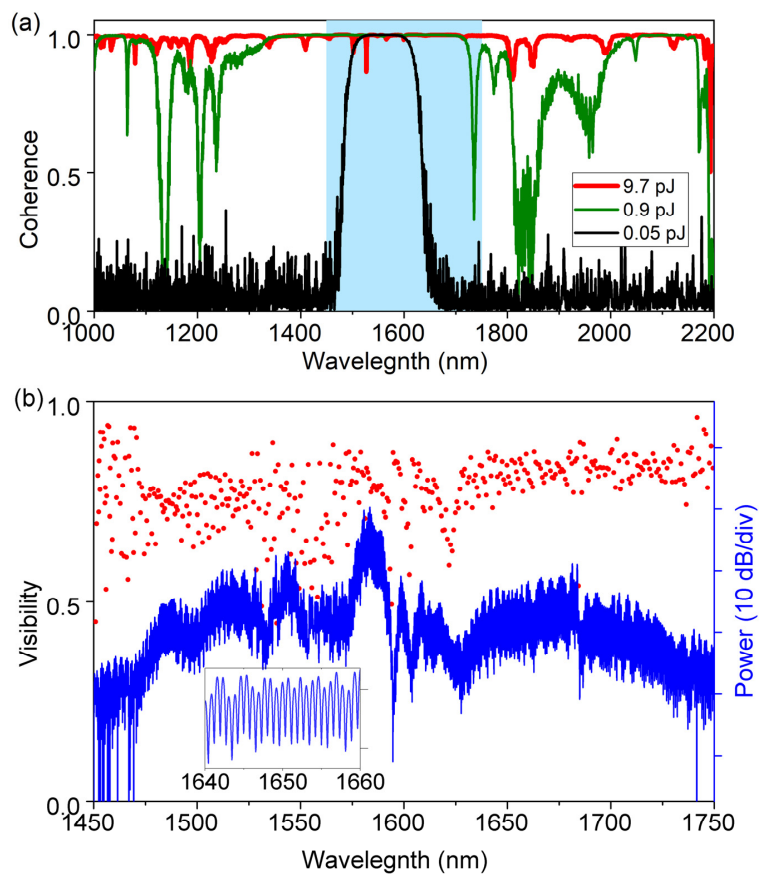

Fig. 5. (a) Simulated coherence for the supercontinuum. (b) Measured spectrum at the output of the interferometer (blue) and the extracted visibility of the spectral fringes (red). Inset shows a magnified range of the spectral fringes.

interference. Moreover, for equal intensities the coherence can be inferred directly from the visibility (see eq. (4). Fig. 5(b) shows the measured output spectrum of the interferometer from $1450 \mathrm{~nm}$ to $1750 \mathrm{~nm}$ (blue curves). The bandwidth of the interferometer is limited by the fiber components which are designed for application in the telecom band. The inset of Fig. 5(b) shows that the fringes have a relatively high contrast (about $10 \mathrm{~dB}$ ), indicating a strong phase coherence of consequent pulses as predicted by the calculation. In Fig. 5(b), the red data points shows the extracted spectral visibility and the majority of the data points are larger than 0.8 indicating a good coherence of the SC, limited to the resolution of the optial spectrum analyzer.

In conclusion, we demonstrated an octave-spaning SCG in a 3mm-long high-confinement AlGaAsOI waveguide. The coherence of the generated supercontinuum has been validated using an interferometry measurement. The waveguide dispersion was engineered to generate two dispersive waves at $f$ and $2 f$ wavelengths. Such dispersive wave generation can also enable octave-spanning Kerr comb generation in high Q microresonators [38]. Similar to the self-referencing demonstrations in AlN [18] and $\mathrm{Si}_{3} \mathrm{~N}_{4}$ [39] platforms, the AlGaAsOI platform also has the potential to realize the $f$-to- $2 f$ self-referencing by generation of a dispersive wave and a second harmonic wave at the same wavelength using a single waveguide if its dispersion is carefully designed. Thanks to the strong $\chi^{(2)}$ and $\chi^{(3)}$ nonlinearities, such a self-referencing process in high-confinement AlGaAsOI waveguide is expected to be more efficient. Besides, the ultra-high device effective nonlinearity of the AlGaAsOI waveguide is also essential for various on-chip pulse compression techniques [40], [41]. This makes it possible to compress pico-second pulses from on-chip mode-locked lasers [42] to femtosecond pulses, which are required for octave-spanning coherent SCG but elusive so far from on-chip lasers. Beneficial to both pulse compression and SCG, the AlGaAsOI waveguides are very promising in realization of a fullyintegrated octave-spanning SC source that can find applications in frequency metrology and frequency synthesis.
Funding. European Research Council (ERC) ELECTRIC project ( 759483) ; Danish National Research Council (DNRF) center SPOC (DNRF123);

Disclosures. The authors declare no conflicts of interest.

\section{References}

1. R. R. Alfano and S. L. Shapiro, Phys. Rev. Lett. 24, 584 (1970).

2. D. J. Jones and Diddams, Science (80-. ). 288, 635 (2000).

3. T. Morioka, K. Mori, and M. Saruwatari, Electron. Lett. 29, 862 (1993).

4. M. J. Thorpe, Science (80-. ). 311, 1595 (2006).

5. A. M. Heidt et al., Opt. Express 19, 13873 (2011).

6. J. M. Dudley, G. Genty, and S. Coen, Rev. Mod. Phys. 78, 1135 (2006).

7. F. Leo, S. Gorza, S. Coen, B. Kuyken, and G. Roelkens, Opt. Lett. 40, 123 (2015).

8. N. Singh et al., Light Sci. Appl. 7, 17131 (2018).

9. Y. Yu et al., Laser Photon. Rev. 8, 792 (2014).

10. J.-É. Tremblay, M. Malinowski, K. A. Richardson, S. Fathpour, and M. C. Wu, Opt. Express 26, 21358 (2018).

11. J. Lu, J. B. Surya, X. Liu, Y. Xu, and H. X. Tang, Opt. Lett. 44, 1492 (2019).

12. M. Yu, B. Desiatov, Y. Okawachi, A. L. Gaeta, and M. Lončar, Opt. Lett. 44, 1222 (2019).

13. R. Halir, Y. Okawachi, J. S. Levy, M. A. Foster, M. Lipson, and A. L. Gaeta, Opt. Lett. 37, 1685 (2012).

14. H. Guo et al., Nat. Photonics 12, 330 (2018).

15. T. Udem, R. Holzwarth, and T. W. Hänsch, Nature 416, 233 (2002).

16. E. Timurdogan, C. V. Poulton, M. J. Byrd, and M. R. Watts, Nat. Photonics 11, 200 (2017).

17. A. Billat, D. Grassani, M. H. P. Pfeiffer, S. Kharitonov, T. J. Kippenberg, and C.-S. Brès, Nat. Commun. 8, 1016 (2017).

18. D. D. Hickstein et al., Phys. Rev. Appl. 8, 014025 (2017).

19. C. Xiong et al., Opt. Express 19, 10462 (2011).

20. E. Stassen, M. Pu, E. Semenova, E. Zavarin, W. Lundin, and K. Yvind, Opt. Lett. 44, 1064 (2019).

21. Y. Zheng et al., Opt. Express 27, 13053 (2019).

22. C. Wang et al., Optica 5, 1438 (2018).

23. U. D. Dave et al., Opt. Lett. 40, 3584 (2015).

24. K. Schneider, P. Welter, Y. Baumgartner, H. Hahn, L. Czornomaz, and P. Seidler, J. Light. Technol. 36, 2994 (2018).

25. J. S. S. Aitchison, D. C. C. Hutchings, J. U. U. Kang, G. I. I. Stegeman, and A. Villeneuve, IEEE J. Quantum Electron. 33, 341 (1997).

26. I. Shoji, T. Kondo, A. Kitamoto, M. Shirane, and R. Ito, J. Opt. Soc. Am. B 14, 2268 (1997).

27. M. Pu, L. Ottaviano, E. Semenova, and K. Yvind, Optica 3, 823 (2016).

28. M. Pu et al., Laser Photon. Rev. 12, 1800111 (2018).

29. L. Chang et al., Laser Photon. Rev. 12, 1800149 (2018).

30. S. May, M. Kues, M. Clerici, and M. Sorel, Opt. Lett. 44, 1339 (2019).

31. H. Hu et al., Nat. Photonics 12, 469 (2018).

32. J. Chiles et al., Optica 6, 1246 (2019).

33. D. T. Spencer et al., Nature 557, 81 (2018).

34. L. Ottaviano, M. Pu, E. Semenova, and K. Yvind, Opt. Lett. 41, 3996 (2016).

35. Y. Zheng, M. Pu, H. K. Sahoo, E. Semenova, and K. Yvind, J. Light. Technol. 37, 868 (2019).

36. M. Pu, L. Liu, H. Ou, K. Yvind, and J. M. Hvam, Opt. Commun. 283, 3678 (2010).

37. G. Agrawal, Nonlinear Fiber Optics. Academic Press, 2013

38. D. T. Spencer et al., Nature 557, 81 (2018).

39. D. R. Carlson et al., Opt. Lett. 42, 2314 (2017).

40. P. Colman et al., Nat. Photonics 4, 862 (2010).

41. D. T. H. Tan, A. M. Agarwal, and L. C. Kimerling, Laser Photon. Rev. 9, 294 (2015).

42. Z. Wang et al., Light Sci. Appl. 6, e16260 (2017). 


\section{Full reference}

[1] R. R. Alfano \& S. L. Shapiro, "Emission in the region 4000 to $7000 \backslash A A$ via four-photon coupling in glass," Phys. Rev. Lett. 24584 (1970).

[2] D. J. Jones \& Diddams, "Carrier-Envelope Phase Control of Femtosecond Mode-Locked Lasers and Direct Optical Frequency Synthesis," Science (80-. ). 288 635-639 (2000).

[3] T. Morioka, K. Mori, \& M. Saruwatari, "More than 100-wavelengthchannel picosecond optical pulse generation from single laser source using supercontinuum in optical fibres," Electron. Lett. 29 862-864 (1993).

[4] M. J. Thorpe, "Broadband Cavity Ringdown Spectroscopy for Sensitive and Rapid Molecular Detection," Science (80-. ). 311 1595-1599 (2006).

[5] A. M. Heidt, J. Rothhardt, A. Hartung, H. Bartelt, E. G. Rohwer, J. Limpert, \& A. Tünnermann, "High quality sub-two cycle pulses from compression of supercontinuum generated in all-normal dispersion photonic crystal fiber," Opt. Express 1913873 (2011).

[6] J. M. Dudley, G. Genty, \& S. Coen, "Supercontinuum generation in photonic crystal fiber," Rev. Mod. Phys. 78 1135-1184 (2006).

[7] F. Leo, S. Gorza, S. Coen, B. Kuyken, \& G. Roelkens, "Coherent supercontinuum generation in a silicon photonic wire in the telecommunication wavelength range," Opt. Lett. 40 123-126 (2015).

[8] N. Singh, M. Xin, D. Vermeulen, K. Shtyrkova, N. Li, P. T. Callahan, E. S. Magden, A. Ruocco, N. Fahrenkopf, C. Baiocco, B. P.-P. Kuo, S. Radic, E. Ippen, F. X. Kärtner, \& M. R. Watts, “Octave-spanning coherent supercontinuum generation in silicon on insulator from $1.06 \mu \mathrm{m}$ to beyond 2.4 um," Light Sci. Appl. 717131 (2018).

[9] Y. Yu, X. Gai, P. Ma, D.-Y. Choi, Z. Yang, R. Wang, S. Debbarma, S. J. Madden, \& B. Luther-Davies, “A broadband, quasi-continuous, midinfrared supercontinuum generated in a chalcogenide glass waveguide," Laser Photon. Rev. 8 792-798 (2014).

[10] J.-É. Tremblay, M. Malinowski, K. A. Richardson, S. Fathpour, \& M. C. $\mathrm{Wu}$, "Picojoule-level octave-spanning supercontinuum generation in chalcogenide waveguides," Opt. Express 2621358 (2018).

[11] J. Lu, J. B. Surya, X. Liu, Y. Xu, \& H. X. Tang, “Octave-spanning supercontinuum generation in nanoscale lithium niobate waveguides," Opt. Lett. 44 1492-1495 (2019).

[12] M. Yu, B. Desiatov, Y. Okawachi, A. L. Gaeta, \& M. Lončar, “Coherent two-octave-spanning supercontinuum generation in lithium-niobate waveguides," Opt. Lett. 44 1222-1225 (2019).

[13] R. Halir, Y. Okawachi, J. S. Levy, M. A. Foster, M. Lipson, \& A. L. Gaeta, "Ultrabroadband supercontinuum generation in a CMOS-compatible platform," Opt. Lett. 37 1685-1687 (2012).

[14] H. Guo, C. Herkommer, A. Billat, D. Grassani, C. Zhang, M. H. P. Pfeiffer, W. Weng, C.-S. Brès, \& T. J. Kippenberg, "Mid-infrared frequency comb via coherent dispersive wave generation in silicon nitride nanophotonic waveguides," Nat. Photonics 12 330-335 (2018).

[15] T. Udem, R. Holzwarth, \& T. W. Hänsch, "Optical frequency metrology," Nature 416 233-237 (2002).

[16] E. Timurdogan, C. V. Poulton, M. J. Byrd, \& M. R. Watts, "Electric field-induced second-order nonlinear optical effects in silicon waveguides," Nat. Photonics 11 200-206 (2017).

[17] A. Billat, D. Grassani, M. H. P. Pfeiffer, S. Kharitonov, T. J. Kippenberg, $\&$ C.-S. Brès, "Large second harmonic generation enhancement in Si3N4 waveguides by all-optically induced quasi-phase-matching," Nat. Commun. 81016 (2017).

[18] D. D. Hickstein, H. Jung, D. R. Carlson, A. Lind, I. Coddington, K. Srinivasan, G. G. Ycas, D. C. Cole, A. Kowligy, C. Fredrick, S. Droste, E. S. Lamb, N. R. Newbury, H. X. Tang, S. A. Diddams, \& S. B. Papp, "Ultrabroadband Supercontinuum Generation and Frequency-Comb Stabilization Using On-Chip Waveguides with Both Cubic and Quadratic Nonlinearities," Phys. Rev. Appl. 8014025 (2017).

[19] C. Xiong, W. Pernice, K. K. Ryu, C. Schuck, K. Y. Fong, T. Palacios, \& H. $X$. Tang, "Integrated GaN photonic circuits on silicon (100) for second harmonic generation," Opt. Express 1910462 (2011).

[20] E. Stassen, M. Pu, E. Semenova, E. Zavarin, W. Lundin, \& K. Yvind, "High-confinement gallium nitride-on-sapphire waveguides for integrated nonlinear photonics," Opt. Lett. 441064 (2019).

[21] Y. Zheng, M. Pu, A. Yi, B. Chang, T. You, K. Huang, A. N. Kamel, M. R. Henriksen, A. A. Jørgensen, X. Ou, \& H. Ou, "High-quality factor, highconfinement microring resonators in $4 \mathrm{H}$-silicon carbide-on-insulator," Opt. Express 2713053 (2019).

[22] C. Wang, C. Langrock, A. Marandi, M. Jankowski, M. Zhang, B. Desiatov, M. M. Fejer, \& M. Lončar, "Ultrahigh-efficiency wavelength conversion in nanophotonic periodically poled lithium niobate waveguides," Optica 51438 (2018).

[23] U. D. Dave, C. Ciret, S.-P. Gorza, S. Combrie, A. De Rossi, F. Raineri, G. Roelkens, \& B. Kuyken, "Dispersive-wave-based octave-spanning supercontinuum generation in InGaP membrane waveguides on a silicon substrate," Opt. Lett. 403584 (2015).

[24] K. Schneider, P. Welter, Y. Baumgartner, H. Hahn, L. Czornomaz, \& P. Seidler, "Gallium Phosphide-on-Silicon Dioxide Photonic Devices," J. Light. Technol. 36 2994-3002 (2018).

[25] J. S. S. Aitchison, D. C. C. Hutchings, J. U. U. Kang, G. I. I. Stegeman, \& A. Villeneuve, "The nonlinear optical properties of AIGaAs at the half band gap," IEEE J. Quantum Electron. 33 341-348 (1997).

[26] I. Shoji, T. Kondo, A. Kitamoto, M. Shirane, \& R. Ito, "Absolute scale of second-order nonlinear-optical coefficients," J. Opt. Soc. Am. B 14 2268 (1997)

[27] M. Pu, L. Ottaviano, E. Semenova, \& K. Yvind, "Efficient frequency comb generation in AlGaAs-on-insulator," Optica 3823 (2016).

[28] M. Pu, H. Hu, L. Ottaviano, E. Semenova, D. Vukovic, L. K. Oxenløwe, \& K. Yvind, "Ultra-Efficient and Broadband Nonlinear AlGaAs-onInsulator Chip for Low-Power Optical Signal Processing," Laser Photon. Rev. 121800111 (2018).

[29] L. Chang, A. Boes, X. Guo, D. T. Spencer, M. J. Kennedy, J. D. Peters, N Volet, J. Chiles, A. Kowligy, N. Nader, D. D. Hickstein, E. J. Stanton, S. A. Diddams, S. B. Papp, \& J. E. Bowers, "Heterogeneously Integrated GaAs Waveguides on Insulator for Efficient Frequency Conversion," Laser Photon. Rev. 121800149 (2018).

[30] S. May, M. Kues, M. Clerici, \& M. Sorel, "Second-harmonic generation in AlGaAs-on-insulator waveguides," Opt. Lett. 441339 (2019).

[31] H. Hu, F. Da Ros, M. Pu, F. Ye, K. Ingerslev, E. Porto da Silva, M. Nooruzzaman, Y. Amma, Y. Sasaki, T. Mizuno, Y. Miyamoto, L. Ottaviano, E. Semenova, P. Guan, D. Zibar, M. Galili, K. Yvind, T. Morioka, \& L. K. Oxenløwe, "Single-source chip-based frequency comb enabling extreme parallel data transmission," Nat. Photonics 12 469-473 (2018)

[32] J. Chiles, N. Nader, E. J. Stanton, D. Herman, G. Moody, J. Zhu, J. Connor Skehan, B. Guha, A. Kowligy, J. T. Gopinath, K. Srinivasan, S. A. Diddams, I. Coddington, N. R. Newbury, J. M. Shainline, S. W. Nam, \& R. P. Mirin, "Multifunctional integrated photonics in the mid-infrared with suspended AlGaAs on silicon," Optica 61246 (2019).

[33] D. T. Spencer, T. Drake, T. C. Briles, J. Stone, L. C. Sinclair, C. Fredrick, Q. Li, D. Westly, B. R. Ilic, A. Bluestone, N. Volet, T. Komljenovic, L. Chang, S. H. Lee, D. Y. Oh, M.-G. Suh, K. Y. Yang, M. H. P. Pfeiffer, T. J. Kippenberg, E. Norberg, L. Theogarajan, K. Vahala, N. R. Newbury, K. Srinivasan, J. E. Bowers, S. A. Diddams, \& S. B. Papp, “An opticalfrequency synthesizer using integrated photonics," Nature 557 81-85 (2018).

[34] L. Ottaviano, M. Pu, E. Semenova, \& K. Yvind, "Low-loss highconfinement waveguides and microring resonators in AlGaAs-oninsulator," Opt. Lett. 413996 (2016).

[35] Y. Zheng, M. Pu, H. K. Sahoo, E. Semenova, \& K. Yvind, "High-QualityFactor AlGaAs-on-Sapphire Microring Resonators," J. Light. Technol. 37 868-874 (2019).

[36] M. Pu, L. Liu, H. Ou, K. Yvind, \& J. M. Hvam, "Ultra-low-loss inverted taper coupler for silicon-on-insulator ridge waveguide," Opt. Commun. 283 3678-3682 (2010).

[37] G. Agrawal, Nonlinear Fiber Optics. Academic Press, 2013.

[38] D. T. Spencer, T. Drake, T. C. Briles, J. Stone, L. C. Sinclair, C. Fredrick, Q. Li, D. Westly, B. R. Ilic, A. Bluestone, N. Volet, T. Komljenovic, L. Chang, S. H. Lee, D. Y. Oh, M.-G. Suh, K. Y. Yang, M. H. P. Pfeiffer, T. J. Kippenberg, E. Norberg, L. Theogarajan, K. Vahala, N. R. Newbury, K. Srinivasan, J. E. Bowers, S. A. Diddams, \& S. B. Papp, “An opticalfrequency synthesizer using integrated photonics," Nature 557 81-85 
(2018).

[39] D. R. Carlson, D. D. Hickstein, A. Lind, S. Droste, D. Westly, N. Nader, I. Coddington, N. R. Newbury, K. Srinivasan, S. A. Diddams, \& S. B. Papp, "Self-referenced frequency combs using high-efficiency silicon-nitride waveguides," Opt. Lett. 422314 (2017).

[40] P. Colman, C. Husko, S. Combrié, I. Sagnes, C. W. Wong, A. De Rossi, S. Combri?, I. Sagnes, C. W. Wong, A. De Rossi, S. Combrié, I. Sagnes, C. W. Wong, A. De Rossi, S. Combri?, I. Sagnes, C. W. Wong, \& A. De Rossi, "Temporal solitons and pulse compression in photonic crystal waveguides," Nat. Photonics 4 862-868 (2010).

[41] D. T. H. Tan, A. M. Agarwal, \& L. C. Kimerling, "Nonlinear photonic waveguides for on-chip optical pulse compression," Laser Photon. Rev. 9 294-308 (2015).

[42] Z. Wang, K. Van Gasse, V. Moskalenko, S. Latkowski, E. Bente, B. Kuyken, \& G. Roelkens, "A III-V-on-Si ultra-dense comb laser," Light Sci. Appl. 6 e16260 (2017).
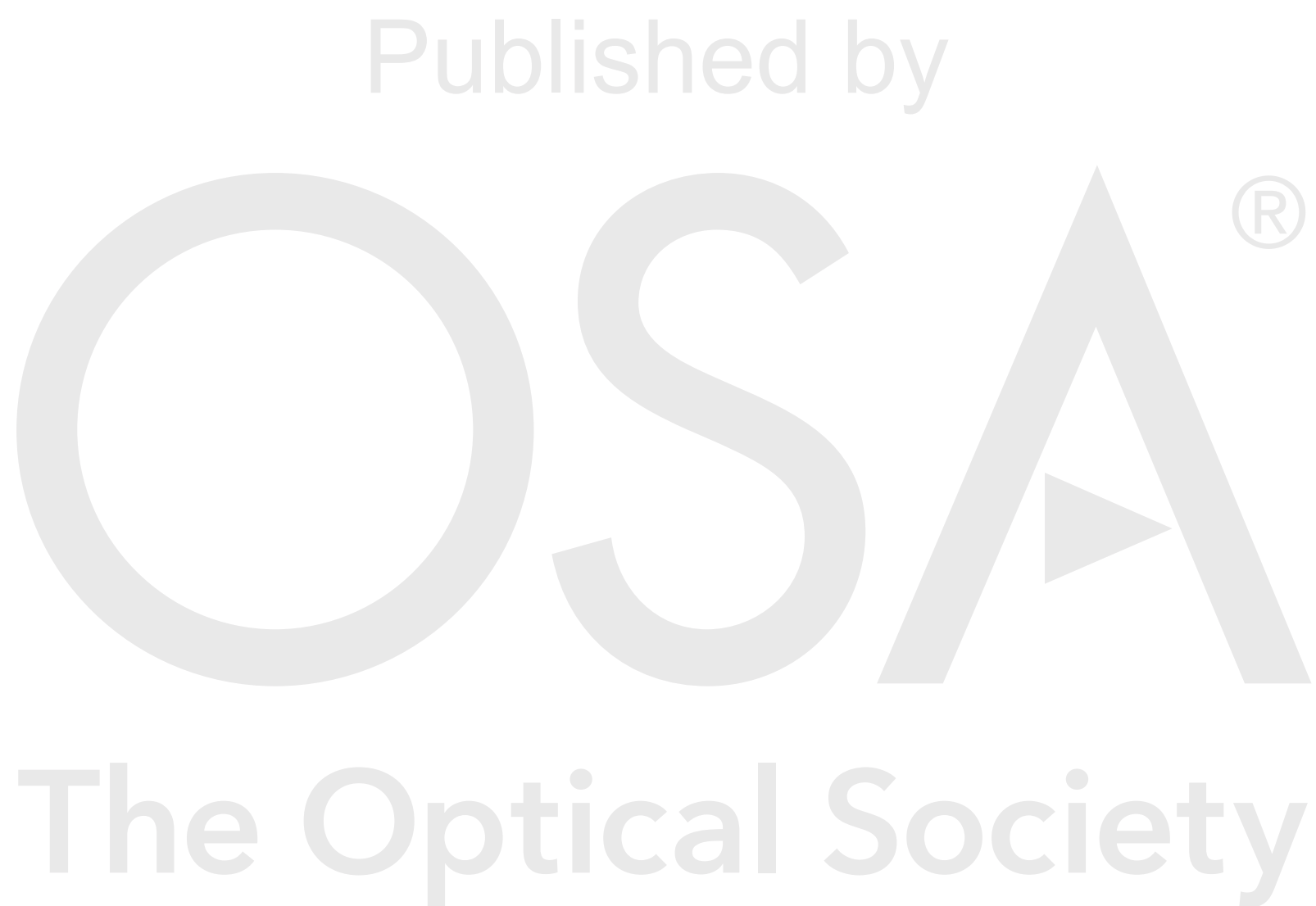